\section{Sample Size: A Conflict in Research Projects of Postgraduate Students}

Sir,

The author has observed many problems with regard to sample size used in research projects of postgraduate students (clinical disciplines), a pre-requisite for postgraduation. The usual study tenure for such research projects is six months in majority or up to a year in few. Many statistical formulae are in vogue to calculate minimum significant sample size required to find outcome of interest/change in response to intervention. These formulae are an appropriate method of computing sample size considering power of study, confidence interval, level of significance etc. into the account. These formulae differ as to the type of study being planned and outcome of interest (qualitative / quanti-tative). ${ }^{1}$

These formulae use values of outcome of interest from previously published studies to compute sample size. This step can be maneuvered by the researchers to get the desired sample size. For example, sample size for a randomised controlled trial, with $5 \%$ significance level ( $80 \%$ power of the study) and putting values of outcome of interest $30 \%$ in one group and $10 \%$ in other (from published data), the sample size will be 60 in each group. ${ }^{2}$ However, if the values of outcome of interest for two groups are $18.5 \%$ to $17 \%,{ }^{3}$ respectively, the sample size would be 9,778 in each group. It means larger the effect size or difference between the groups, smaller the sample size is required to detect and vice versa. It depends upon the researchers, which study they are referring to for sample size calculation.

The author has observed various sample sizes such as 90 choledochal cysts in 6 months, 200 colostomies for anorectal malformation in 6 months, 500 laparoscopic appendicectomies in 1 year, and so on. It is quite obvious that this sample size cannot be recruited in a single unit within these timeframes. But unfortunately, research projects with similar sample size and study duration get approved and even thesis/dissertations based on these sample size/duration get through formal scrutiny. This creates various doubts about integrity of research and scientific evidence these studies would produce. Many projects are based on diseases, which are quite rare in occurrence. Thus researchers may not be able to complete the research within stipulated time period. Moreover, sample size formulae do not consider study duration, while calculating sample size. In these instances, possibility of fabrication or falsification of data may not really be ruled out.

To circumvent these problems, the author suggests some alternative ways of dealing with sample size calculation:

A. Literature-based sample size: The recent literature can be reviewed on the relevant patient population/ outcome of interest and an average sample size and average study tenure of 5-10 most recent studies can be calculated and may be used as sample size for the desired study tenure. This will give an exact idea of number of patients related to a particular disease/ outcome with respect to study duration. However, the author can also selectively choose 5 or 10 small studies and take the average of those samples which ultimately will also be small. Moreover, any study with very large sample size can skew the average in upper side; and opposite can happen with smaller size studies. Lastly, this method of taking averages might not be scientifically justified as well, when writing research paper on a later stage; although selecting studies with effect size of one's choice for sample size collection, is itself a question on scientific value of sample size collection formulae.

B. Multi-centric studies: The projects involving rare disorders can be performed by recruiting patients from multi-centres catering similar population. This will also increase its authenticity; but involves getting approval from multiple institutional review boards and requires extra logistics for data collection.

C. Pilot projects: The students may be allowed to conduct study with small sample size for requirement of postgraduation. But, this would not produce meaningful data and evidence.

D. Interim analysis: The study should continue to recruit participants until the desired sample size is achieved. However, the postgraduate student may be allowed to present interim analysis for the purpose of exit examination. The duration of interim analysis may be debated. The researcher will complete the research afterwards and supervisor may be appointed as custodian of the research project for its completion. The study is only allowed to publish after it gets a completion certificate from the supervisor/institution. A true data in interim analysis is far better than a complete study with falsified/fabricated data.

These are the few suggested ways that may help the postgraduate students in research as requirement for the award of postgradation degrees.

CONFLICT OF INTEREST:

Author declared no conflict of interest. 


\section{AUTHOR'S CONTRIBUTION:}

MBM: Involved in concept, design, draft, literature review and final approval of all versions of the manuscript.

\section{REFERENCES}

1. Charan J, Biswas T. How to calculate sample size for different study designs in medical research?. Indian $\mathrm{J}$ Psychol Med 2013; 35:121.

2. Surgical audit and research. In: Williams NS, Bulstrode CJ. K, O'Connell PR (eds). Bailey \& Love's short practice of surgery. 25th Edn. Hodder Arnold: London; 2008; pp. 164.

3. Katkhouda N, Mason RJ, Towfigh S, Gevorgyan A, Essani R.
Laparoscopic versus open appendectomy: A prospective randomized double-blind study. Annals Surg 2005; 242:439.

\section{Bilal Mirza}

Department of Pediatric Surgery, The Children's Hospital and The Institute of Child Health, Lahore, Pakistan

Correspondence to: Dr. M. Bilal Mirza, Department of Pediatric Surgery, The Children's Hospital and The Institute of Child Health, Lahore, Pakistan

Email: blmirza@gmail.com

Received: December 29, 2018; Revise: March 17, 2019; Accepted: March 26, 2019.

\section{...........}

